\title{
Impact of Madden-Julian Oscillation on North Indian Ocean Cyclone
}

\section{Intensity}

\author{
MM Ali ${ }^{1,2, *}$, UN Tanusha ${ }^{2}$, C. Purna Chand ${ }^{2}$, B. Himasri ${ }^{2}$, M. A. Bourassa ${ }^{1}$, Y. Zheng $^{1}$ \\ ${ }^{1}$ Center for Ocean-Atmospheric Prediction Studies, Florida State University, USA \\ ${ }^{2}$ Andhra Pradesh State Disaster Management Authority, India \\ *Corresponding author: mmali110@gmail.com
}

\begin{abstract}
The influence of the Madden - Julian Oscillation (MJO) on the intensity of the Tropical Cyclones in the North Indian Ocean is investigated through a machine learning algorithm using cyclone data from the Joint Typhoon Warning Centre and MJO from the Bureau of Meteorology, Australia during 1974-2019. The scatter index varies from 0.45 for depressions to 0.03 for the super cyclonic storms indicating that the MJO index is another parameter that should be investigated in cyclone studies.
\end{abstract}

Keywords: MJO; Cyclone; winds; Machine learning.

\section{Introduction:}

The Madden - Julian Oscillation (MJO), the dominant sub-seasonal variability prominently represents the intraseasonal variability in the tropics (Tian \& Waliser, 2014) and bridges weather and climate (Wu et al., 2016; Zhang et al., 2020). MJO has a spatial structure of zonal wavenumber with large scale signals in atmospheric circulation, deep convection propagating eastwards with a speed of $5 \mathrm{~m} / \mathrm{s}$ from the Indian Ocean to Pacific Ocean (Liu et al., 2016). The MJO influences weather and climate variability, Arctic Oscillations and North Atlantic Oscillations (Wu et al., 2016), El Nino Southern Oscillations (Kikuchi et al., 2012; Wu et al., 2016), onset of monsoon and monsoon breaks over East Asia (Liu et al., 2016; Wu et al., 2016; 
Vitart, 2009; Wu et al., 2016), barotropic sea level variability (Rohith et al., 2019) and Tropical Cyclones (TCs:Tsuboi \& Takemi, 2014; Belanger, 2012; Krishnamohan et al., 2012; Vitart, 2009).

Even though cyclones cannot form during the monsoon season, low pressure areas, depressions and deep depressions are very common in the Indian Ocean, which bring significant amount of rainfall during this season. TCs are one of the dangerous meteorological disasters causing deaths, damaging agricultural crops and economics along the coasts. Hence, this phenomenon and its predictability are worth investigating. Disaster management authorities look for an effective prediction of cyclone track and intensity for the planning of mitigations processes. (Sinha et al., 2014; Willoughby et al., 2007). Strong winds in a TC create economic damage in the coastal and inland areas. Wind speed, along with storm track and size, also helps predict storm surge, which is the most devastating component of the cyclones for coastal India, particularly in regions that have highly varying bathymetry.

While many factors influence the intensity of a cyclone, in this paper we study the impact of MJO alone without considering the other factors. Cyclone information available from Joint Typhoon Warning Centre (JTWC) during 1972-2019 has been used here to study the impact of MJO on cyclone intensity represented by the one minute sustained maximum wind magnitude, which is a reasonable diagnostic for wind damage. Girishkumar et al., (2015) studied the impact of MJO on the genesis of the North Indian Ocean (NIO) cyclones. Tsuboi \& Takemi (2014) investigated the interannual relationship between the MJO activity and the TC genesis in the Indian Ocean. The combined impact of ENSO and MJO on the rapid intensification of TCs in the Bay of Bengal was investigated by Girishkumar et al. (2015). Herein, The MJO components, such as real-time multivariate MJO (RMM) indices from which 
the amplitude and phase are estimated are used in a machine learning algorithm to study the impact of MJO on cyclone wind speeds.

Artificial neural network (ANN) is one of the popular and powerful machine learning algorithms for predicting output from a trained input-output relationship. It works on a principle similar to the neurons in a human brain, consisting of interconnected assembly of models (Swain et al., 2006). The ANN models are generally used in a standalone or in a statistical analysis in a complement mode. These models have been used in many oceanographic, meteorological and satellite parameter retrieval studies(Sharma et al., 2013b; Ali et al., 2004, Tolman et al., 2005, Jain and Ali 2006, Swain et al., 2006, Jain et al., 2007, Ali et al., 2012a, b, c, Liu et al., 1997, Ali et al., 2007, Sharma and Ali., 2012,; Krasnopolsky and Schiller, 2003, Ali et al., 2015, Ali et al., 2016). It is imperative to normalize the input and output variables to vary between 0 and 1 in an ANN analysis

The three data sets required for an ANN model are, (i)for training, (ii) for verification and the (iii) for validation. The model is trained with the first dataset., the model is tested for any shortcomings using the second data set. The third dataset, which is held back and not used in developing the model, is used to compare the predicted data with observations and in statistical parameters estimation. Some of the well known models of ANN are Multi-Layer Perceptron (MLP), Radial basis functions (RBF), and Conjugate Gradient Descent models (Jain et al., 2007; Broomhead and Lowe, 1988; Moody and Darkin, 1989; Haykin, 2002). Here, we used the MLP method. 


\section{Data and Methodology}

\subsection{Data}

In this study, we used wind magnitudes of the NIO cyclones during 1974-2019 from JTWC provided by the International Best Track Archive for Climate Stewardship (IBTrACS; Knapp et al., 2010: https://www.ncep.noaa.gov/data/international-best-track-archive-for-climatestewardship-ibtracs/v04r00/access/csv/). Latitude, longitude, surface pressure and wind speed of cyclones are provide in the JTWC dataset. Out of the 3 and 6 hourly interval data, we used 6-hour interval data in this study. After discarding cyclone positions that are at irregular intervals we used 239 cyclones. Daily values of MJO data for the same period were taken from the Bureau of methodology, Australia. These data contain RMM1 and RMM2, its phase (determined from signed values of RMM1 and RMM2) and amplitude (computed as $\left.\sqrt{R M M 1^{2}+R M M 2^{2}}\right)$ (Wheeler and Hendon 2004).

\subsection{Methodology}

These MJO values have been collocated with cyclone parameters. Since the cyclone information is generally available four times a day at $0,6,12$ and 18 hours but the MJO values are available only once a day, daily MJO values have been repeated for these four observations of a cyclone. This type of collocation is justified because MJO is of much longer duration compared the changes in cyclone intensities. These collocated observations have year, month, date, hour, cyclone number, latitude, longitude and wind speed from JTWC and year, month, date RMM1, RMM2, phase and amplitude from the Bureau of Meteorology, Australia. From these collocated observations, 7 datasets have been created for different categories of the cyclonic systems following Mohapatra et al. (2019). The numbers in parenthesis represent the number of events. If a cyclone has four observations a day as described earlier, the number of events is 4 . 
1. All the cyclonic systems which are Depressions (D) and above (231)

2. Deep Depressions (DD) and above (229)

3. Cyclonic Storms (CS) and above (222)

4. Severe Cyclonic Storms (SCS) and above (130)

5. Very Severe Cyclonic Storms (VSCS) and above (74)

6. Extremely Severe Cyclonic Storms (ESCS) and above (34)

7. Super Cyclonic Storms (SUCS) (15)

While 222 CS events and above were formed in the entire study period, SUCS were only 15.

ANN analysis has been carried out on these 7 types of datasets. The data were randomly selected for training (70\%), , for verification (15\%) and for validation (15\%) This random selection avoids any bias that might have crept if selected on any specific criteria. Wind speed is used as a proxy for the intensity of the cyclones.

\section{Results}

The ANN model was run with wind speed from JTWC as the predictand and month, date, RMM1, RMM2, Phase and amplitude from the Bureau of meteorology as the predictors. The R- squared, F- statistics, and p-values between the predictand and the predictors are given in Table 1.

Table 1: The statistical parameters between the predictand (wind speed) and the predictors (month, date, RMM1, RMM2, phase and amplitude).

\begin{tabular}{|c|c|c|c|}
\hline Parameter & R-squared & F-statistics & p-value \\
\hline RMM2 & 0.058 & 42.45 & 0.00 \\
\hline Month & 0.041 & 34.21 & 0.00 \\
\hline Amplitude & 0.040 & 34.03 & 0.00 \\
\hline Day & 0.017 & 12.17 & 0.00 \\
\hline RMM1 & 0.014 & 11.84 & 0.00 \\
\hline Phase & 0.010 & 9.95 & 0.00 \\
\hline
\end{tabular}


The R-squared values are very small because the cyclone wind magnitudes are controlled by many other metrological factors other than the MJO indices alone. RMM2 has the highest R-squared value, followed by the month. Kikuchi et al., 2012 concluded that intraseasonal oscillations (ISO) at any time of the year can be expressed in terms of MJO mode during December to April and boreal summer ISO during June-October. The histogram of the number of cyclonic systems formed in different months during the study period is shown in Fig.1. If a system was present in two months, it is considered for both the months. Most of the systems are present during April-June or from September to December, peaking in November. This could be one of the reasons for the month having the second-highest R-squared value.

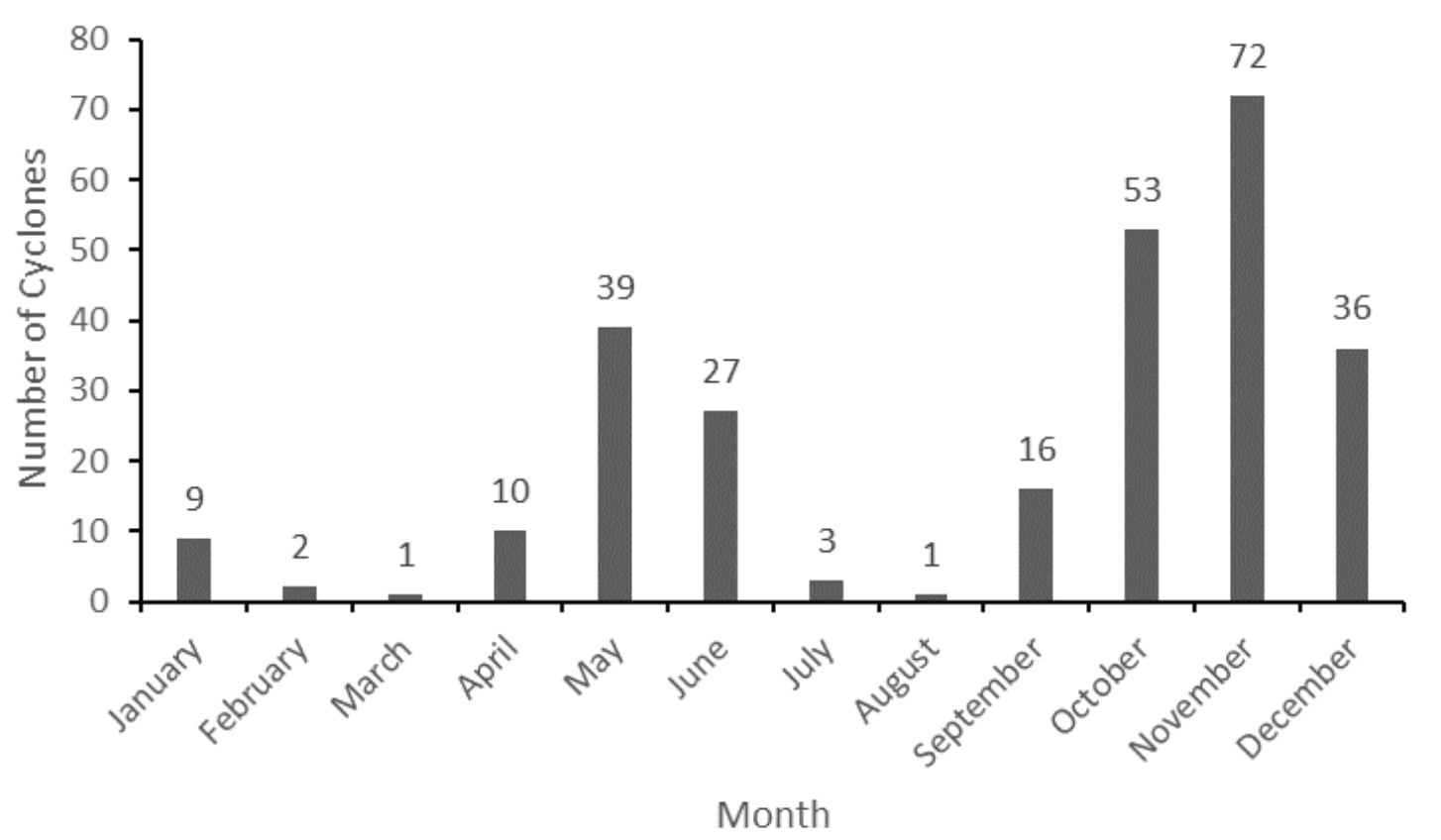

Fig 1: Histogram of the number of cyclonic systems of all categories formed in different months during 1974-2019. The numbers on the bars represent the number of systems in that month.

The percentage of the deviations calculated from the histograms falling between $-80 \% \mathrm{~m} / \mathrm{s}$ to $80 \% \mathrm{~m} / \mathrm{s}$ for the validation dataset for all these 7 categories is shown in Table 2 . While about 
$80 \%$ of the estimations under the validation category fall between $-20 \% \mathrm{~m} / \mathrm{s}$ to $20 \% \mathrm{~m} / \mathrm{s}$ for $\mathrm{D}$, DD, CS, SCS and VSCS, 70\% observations fall for ESCS and only 37\% for SUCS. Most of the estimations for SUCS fall under $20-40 \% \mathrm{~m} / \mathrm{s}$. This could be because the points for training and validation are less for SUCS compared to the other cyclone categories due to which training could not be properly calculated. The statistics for ESCS and SUCS have too large a reduction in percentage change to support the suggestion that this result could simply indicate that rapid changes in intensity are relatively rare and that a similar absolute change in intensity results in a smaller percentage change for stronger storms. We later confirm that this simple explanation is incorrect. While this consideration likely contributes to the reduction in percentage change with stronger storms, the explanation for SUCS is likely that such storms can be relatively stable, particularly when the storm becomes annular. Under these conditions, the storms have relatively small absolute changes in intensity. Furthermore, the strongest storms almost always form under near-ideal conditions for a strong storm, and hence are less likely to lose strength interacting with phenomena that inhibit the development of storms (other than encounters with land).

Table 2: Percentage of Deviations between the estimated and observed values for validation dataset in different Categories. The number in the parenthesis represents the total number of observations during all the 4 observation times (at 00, 06, 12, $18 \mathrm{hrs).}$

\begin{tabular}{|c|c|c|c|c|c|c|c|}
\hline $\begin{array}{c}\text { Percent } \\
\text { change (\%) }\end{array}$ & D (720) & DD (505) & CS (394) & SCS (180) & VSCS (94) & ESCS (39) & SUCS (8) \\
\hline $\mathbf{- 8 0}-\mathbf{- 6 0}$ & 0.14 & 0.00 & 0.00 & 0.00 & 0.00 & 0.00 & 0.00 \\
\hline $\mathbf{- 6 0}-\mathbf{- 4 0}$ & 0.28 & 0.00 & 0.00 & 0.00 & 0.00 & 0.00 & 0.00 \\
\hline $\mathbf{- 4 0}-\mathbf{- 2 0}$ & 8.89 & 9.50 & 8.88 & 6.67 & 8.51 & 17.95 & 0.00 \\
\hline $\mathbf{- 2 0}-\mathbf{0}$ & 47.22 & 50.89 & 52.28 & 50.56 & 43.62 & 25.64 & 12.50 \\
\hline $\mathbf{0}-\mathbf{2 0}$ & 33.19 & 30.69 & 29.19 & 30.00 & 29.79 & 33.33 & 25.00 \\
\hline $\mathbf{2 0}-\mathbf{4 0}$ & 7.22 & 6.73 & 8.12 & 11.11 & 14.89 & 17.95 & 62.50 \\
\hline $\mathbf{4 0}-\mathbf{6 0}$ & 1.81 & 0.99 & 0.51 & 0.56 & 1.06 & 0.00 & 0.00 \\
\hline $\mathbf{6 0}-\mathbf{8 0}$ & 0.97 & 1.19 & 1.02 & 1.11 & 2.13 & 5.13 & 0.00 \\
\hline
\end{tabular}



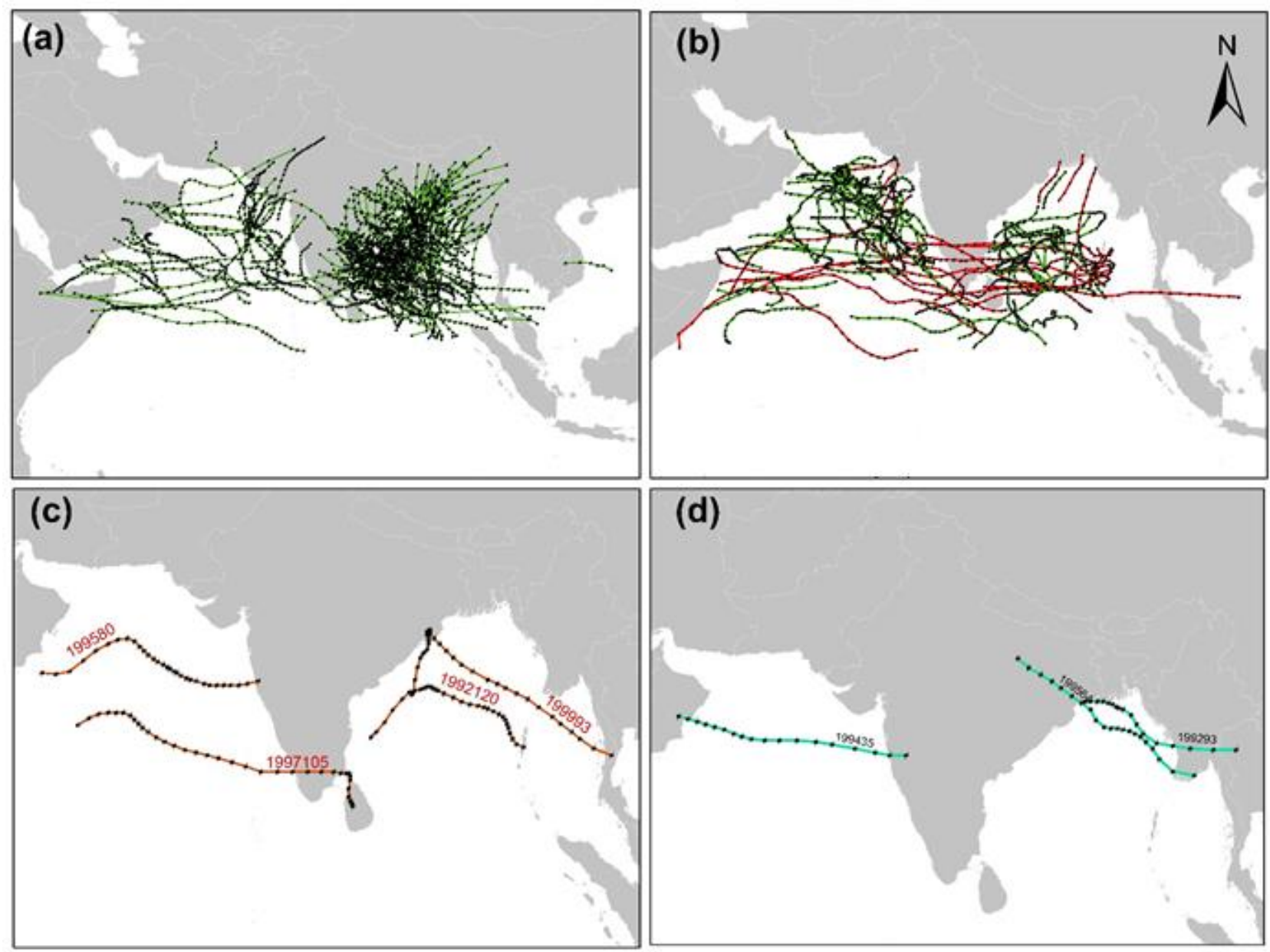

Fig 2: Cyclone tracks that have (a) formed over the sea and dissipated over the land (No. of cyclones 142), (b) formed over the sea and dissipated over the sea itself without crossing the land (No. of cyclones 60; red in colour), formed over the sea and dissipated over the sea after crossing the land (No. of cyclones 22; green in colour), (c) formed over land and dissipated over sea and (d) formed over land and dissipated over land.

The cyclones during the study period can be divided into

1. Those that have formed over the ocean and dissipated over the land (Fig 2a).

2. Those that have formed over the ocean and dissipated over the oceans with and without crossing the land (Fig 2b). 
3. Those that formed over the land moved to the ocean and dissipated (fig 2c) and

4. Those that have formed over the land moved to the land after passing over oceans and dissipated over the land. The number of cyclones of these four types are 142, 82, 4 and 3 , respectively.

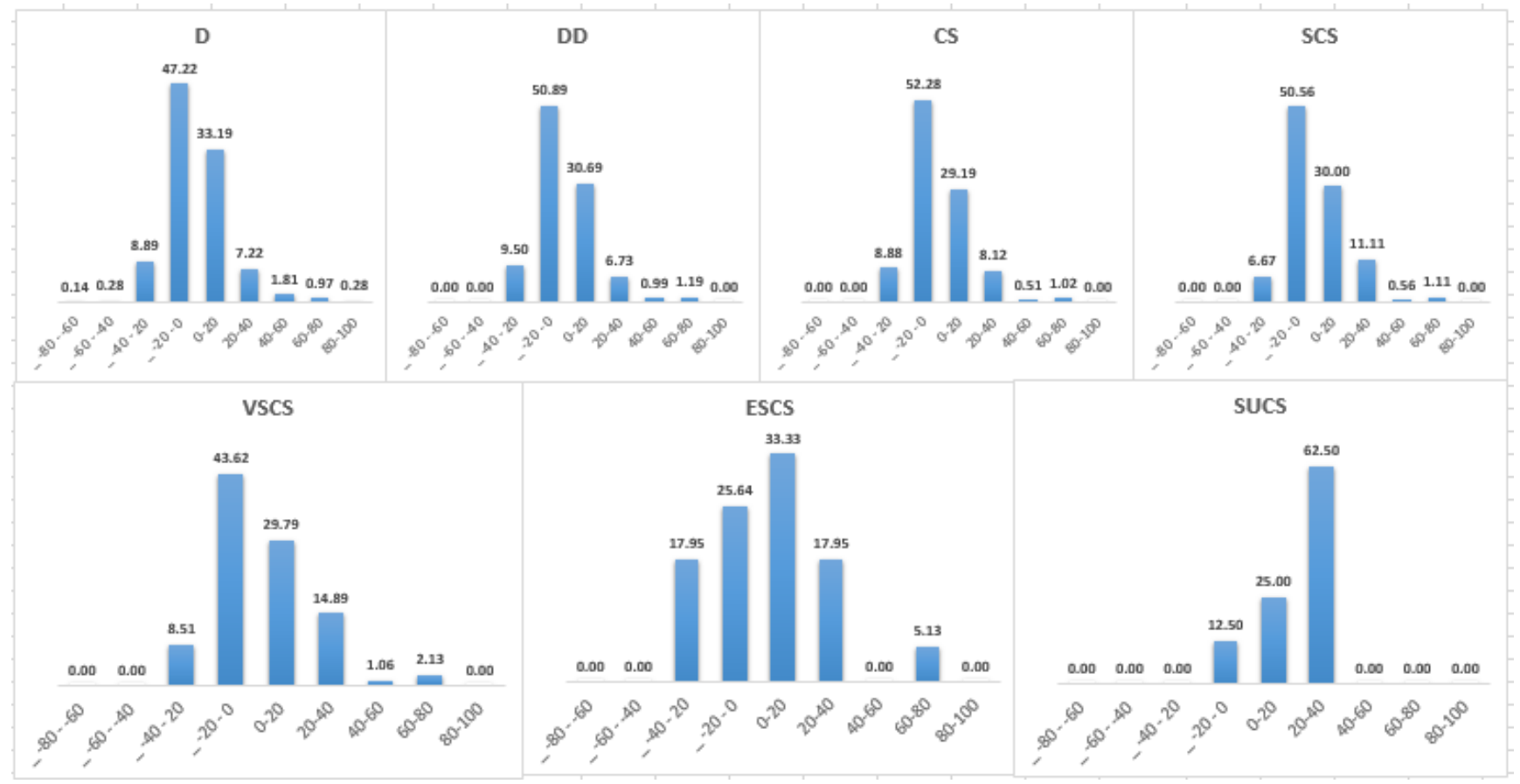

Fig 3: Percentage of Deviations for validation datasets divided into Categories of $\mathrm{D}, \mathrm{DD}, \mathrm{CS}$, SCD, VSCS, ESCS and SUCS.

Percentage deviations between the observed and MJO-based ANN estimated wind speeds are shown in Figure 3. RMSE (Root Mean Square Error) and SI (Scatter Index) of the wind magnitudes for the entire dataset and the validation dataset for the 7 categories of cyclones is presented in Table 3. Almost similar values of RMSE and SI for the entire and validation datasets indicate that the random selection of the data sets for the ANN analysis equally covers all the ranges of MJO and wind speeds. The RMSE varies from $4.38 \mathrm{~m} / \mathrm{sec}$ for the SUCS to $19.25 \mathrm{~m} / \mathrm{sec}$ for DD, for the validation dataset. While RMSE is more useful for emergency managers, the SI estimates defined as RMSE normalized to the observed mean value, are one 
of the best statistical parameters to judge the skill of the estimations. The SI is highest for D (0.45) and gradually decreases as the cyclone intensity categories increase and it is the least (0.03) for the SUCS. SI of much less than 1 indicates that the MJO parameters play a significant role in predicting cyclone intensity. Relatively lower values of RMSE and SI for the entire and validation datasets indicate that the MJO plays a major role for the cyclones with higher intensities.

Table 3: Statistical parameters for RMSE and SI for all the categories for the entire and validation dataset alone.

\begin{tabular}{|c|c|c|c|c|c|c|}
\hline & \multicolumn{3}{|c|}{ All } & \multicolumn{3}{c|}{ Validation } \\
\cline { 2 - 7 } & Count & RMSE & SI & Count & RMSE & SI \\
\hline D & 4805 & 18.26 & 0.45 & 720 & 18.38 & 0.45 \\
\hline DD & 3369 & 17.66 & 0.38 & 505 & 19.25 & 0.41 \\
\hline CS & 2627 & 18.07 & 0.34 & 394 & 19.16 & 0.36 \\
\hline SCS & 1201 & 17.19 & 0.24 & 180 & 17.08 & 0.24 \\
\hline VSCS & 629 & 15.14 & 0.17 & 94 & 13.66 & 0.16 \\
\hline ESCS & 261 & 9.87 & 0.09 & 39 & 10.22 & 0.09 \\
\hline SUCS & 59 & 5.69 & 0.04 & 8 & 4.38 & 0.03 \\
\hline
\end{tabular}

The lower SI values indicate that the MJO-based ANN forecast skill improves with the increasing intensity of cyclonic system categories. This result implies that MJO parameters have more impact on more intense cyclones.

Lafleur et al. (2015) discussed the active role played by MJO amplitudes on cyclone intensities. As discussed by (Lafleur et al., 2015), we divided the dataset into 4 categories of (i) all the amplitudes, (ii) amplitudes $1-1.5$, (iii) amplitudes $1.5-2.0$ and (iv) amplitudes $>2.0$. The SI decreases with increasing amplitude for all categories of the cyclonic systems showing the important role played by amplitude in clone intensity prediction. Wheeler \& Hendon (2004) observed MJO be more active when the amplitude is $>2.0$. However, they did not study its influence on different types of cyclone categories, as is done herein. Besides the SI decreasing 
with increasing amplitude, it also decreases with the increasing intensity of the cyclonic systems. This implies that MJO influences more for high intensity cyclones. We studied the influence of the amplitude on cyclone intensity for (i) all phases together and (ii) for phases $1,2,3,4$ covering the Indian Ocean and the two adjoining phases. In addition, all the phases have been further divided in to different amplitudes as (i) all amplitudes, (ii) amplitudes $>=1$, (iii) $>=1.5$ and (iv) $>=2$. The influence of MJO phases and amplitudes, thus categorized, on the wind speed of the validation data set is given in Table 4. We did not compute SI for ESCS and SUCS as the number of points is too small to meaningfully compute SI when the phases are divided into different categories. The SI is least for amplitudes $>2$ which implies that the impact of MJO on cyclone intensity is more for greater amplitudes.

Table 4: Influence of the amplitude of MJO on estimated wind speed scatter index (SI) for all the categories of the cyclonic systems for (i) all amplitudes, (ii) amplitudes $>=1$, (iii) $>=1.5$ and (iv) >=2 separately for (i) all phases and (ii) phases $1,2,3,4$ for the validation dataset. The number of points for each category is also provided.

The number of cyclones formed when the MJO index in phases 1, 2, 3, 4 is 155 while that formed in phases 5, 6, 7 or 8 is 76. This concludes that when the MJO is in the Indian Ocean phases of 2 or 3 and the two adjoining phases of 1 or 4 the conditions are more conducive to the formation of cyclones. 


\section{Conclusion}

Statistical analysis through a machine learning process indicates that MJO index has an influence on the intensity of the cyclones in the north Indian Ocean. The deviations between the wind speeds estimated using the month, date, RMM1, RMM2, amplitude and phase of the MJO and the actual observations falls between -20 mps to +20 mps for $80 \%$ of the cases for D, DD, CS, SCS, and VCCS categories of the cyclone. However, only $70 \%$ and $37 \%$ of ESCS and SUCS respectively fall under this range. The SI of the validation dataset reduces from 0.45 for D to 0.03 for SUCS implying that MJO has more influence on the cyclones with more intensity. Both MJO phase and amplitude influence the cyclone intensity. The cyclonic systems formed when the MJO is in phases 1, 2, 3 or 4 are double those formed during the 5, 6, 7 or 8 phases. The influence of MJO amplitude on wind speeds increases with increasing amplitude. Here, we statistically studied the influence of MJO alone on cyclone intensities.

\section{Acknowledgments:}

The authors thank their respective organizations for the support and encouragement. Wind magnitudes of the NIO cyclones were obtained from the International Best Track Archive for Climate Stewardship (https://www.ncep.noaa.gov/data/international-best-track-archive-forclimate-stewardship-ibtracs/v04r00/access/csv/). Information on MJO was available at www.bom.gov.au/climate/mjo/graphics/rmm.74toRealtime.txt. The authors thank both the organisations for the data.

The authors have no conflict of interest. 


\section{References}

Belanger, J. I. (2012). Predictability and prediction of tropical cyclones on daily to interannual time scales. August, 176.

Girishkumar, M. S., Suprit, K., Vishnu, S., Prakash, V. P. T., \& Ravichandran, M. (2015). The role of ENSO and MJO on rapid intensification of tropical cyclones in the Bay of Bengal during October-December. Theoretical and Applied Climatology, 120(3-4), 797-810.

Jain, S., Ali, M. M., \& Sen, P. N. (2007). Estimation of sonic layer depth from surface parameters. Geophysical Research Letters, 34(17).

Kikuchi, K., Wang, B., \& Kajikawa, Y. (2012). Bimodal representation of the tropical intraseasonal oscillation. Climate Dynamics, 38(9-10), 1989-2000.

Krishnamohan, K. S., Mohanakumar, K., \& Joseph, P. V. (2012). The influence of MaddenJulian Oscillation in the genesis of North Indian Ocean tropical cyclones. Theoretical and Applied Climatology, 109(1-2), 271-282.

Lafleur, D. M., Barrett, B. S., \& Henderson, G. R. (2015). Some climatological aspects of the Madden-Julian oscillation (MJO). Journal of Climate, 28(15), 6039-6053.

Liu, P., Zhang, Q., Zhang, C., Zhu, Y., Khairoutdinov, M., Kim, H. M., Schumacher, C., \& Zhang, M. (2016). A revised real-time multivariate MJO index. Monthly Weather Review, 144(2), 627-642.

Mohapatra, Mrutyunjay., and Monica Sharma. "Cyclone warning services in India during recent years: A review." Mausam 70.4 (2019): 635-666.

Rohith, B., Paul, A., Durand, F., Testut, L., Prerna, S., Afroosa, M., Ramakrishna, S. S. V. S., \& Shenoi, S. S. C. (2019). Basin-wide sea level coherency in the tropical Indian Ocean driven 
by Madden-Julian Oscillation. Nature Communications, 10(1), 1-9.

Sharma, N., Ali, M. M., Knaff, J. A., \& Chand, P. (2013a). A soft-computing cyclone intensity prediction scheme for the Western North Pacific Ocean. Atmospheric Science Letters, 14(3), $187-192$.

Sharma, N., Ali, M. M., Knaff, J. A., \& Chand, P. (2013b). A soft-computing cyclone intensity prediction scheme for the Western North Pacific Ocean. Atmospheric Science Letters, 14(3), $187-192$.

Tian, B., \& Waliser, D. (2014). Madden-julian oscillation (MJO). Encyclopedia of Earth Sciences Series, 349-358.

Tsuboi, A., \& Takemi, T. (2014). The interannual relationship between MJO activity and tropical cyclone genesis in the Indian Ocean. Geoscience Letters, 1(1), 1-6.

Vitart, F. (2009). Impact of the Madden Julian Oscillation on tropical storms and risk of landfall in the ECMWF forecast system. Geophysical Research Letters, 36(15), 1-6.

Wheeler, M. C., \& Hendon, H. H. (2004). An all-season real-time multivariate MJO index: Development of an index for monitoring and prediction. Monthly Weather Review, 132(8), $1917-1932$.

Wu, J., Ren, H. L., Zuo, J., Zhao, C., Chen, L., \& Li, Q. (2016). MJO prediction skill, predictability, and teleconnection impacts in the Beijing Climate Center Atmospheric General Circulation Model. Dynamics of Atmospheres and Oceans, 75, 78-90.

Zhang, C., Adames, F., Khouider, B., Wang, B., \& Yang, D. (2020). Four Theories of the Madden-Julian Oscillation. Reviews of Geophysics, 58(3). 